\title{
Canine Model for Selective and Superselective Cerebral Intra-Arterial Therapy Testing
}

\author{
Kevin M. Camstra, MS ${ }^{1 \star}$, Visish M. Srinivasan, $\mathrm{MD}^{1}$, Dalis Collins, DVM ${ }^{2,4}$, Stephen Chen, $\mathrm{MD}^{3}$, \\ Peter Kan, $\mathrm{MD}, \mathrm{MPH}^{1}$, Jeremiah Johnson, $\mathrm{MD}^{1 \star}$ \\ ${ }^{1}$ Department of Neurosurgery, Baylor College of Medicine, Houston, TX, USA \\ ${ }^{2}$ Department of Surgery, Baylor College of Medicine, Houston, TX, USA \\ ${ }^{3}$ Department of Radiology, Baylor College of Medicine, Houston, TX, USA \\ ${ }^{4}$ Center for Comparative Medicine, Baylor College of Medicine, Houston, TX, USA
}

Purpose: With advancing endovascular technology and increasing interest in minimally invasive intra-arterial therapies such as stem cell and chemotherapy for cerebral disease, the establishment of a translational model with cerebral circulation accessible to microcatheters is needed. We report our experience catheterizing canine cerebral circulation with microcatheters, present high-resolution angiographic images of the canine vascular anatomy, describe arterial branch flow patterns and provide measurements of canine arterial conduits.

Materials and Methods: Angiograms were performed on 10 intact purpose-bred hounds. Angiography, measurements of arterial conduits and catheterization information for intracranial arterial branches were obtained.

Results: Selective and superselective cerebral angiography was successful in all subjects. Relevant arterial mean diameters include the femoral $(4.64 \mathrm{~mm})$, aorta $(9.38 \mathrm{~mm})$, external carotid $(3.65 \mathrm{~mm})$, internal carotid arteries $(1.6 \mathrm{~mm})$, vertebrobasilar system and Circle of Willis branches. Catheterization of the Circle of Willis was achieved via the posterior circulation in all subjects tested $(n=3)$ and the use of flow directed microcatheters resulted in reduced arterial tree deformation and improved superselection of intracranial vessels. Catheterization of the intracranial circulation was attempted but not achieved via the internal carotid artery $(n=7)$ due to its tortuosity and subsequent catheter related vasospasm.

Conclusion: The canine cerebral vasculature is posterior circulation dominant. Anterior circulation angiography is achievable via the internal carotid artery, but direct cerebral arterial access is best achieved via the posterior circulation using flow-directed microcatheters. It is feasible to deliver intra-arterial therapies to selective vascular territories within the canine cerebral circulation, thus making it a viable animal model for testing novel intra-arterial cerebral treatments.

Key Words: Angiography; Animal model; Intraarterial delivery; Neurovascular

\section{Correspondence to: Jeremiah Johnson, MD Department of Neurosurgery, Baylor College of Medicine, 7200 Cambridge, Suite 9A, Houston, TX 77030, USA \\ Tel: +1-713-798-4696 \\ Fax: +1-713-798-1216 \\ E-mail: jeremiah.johnson@bcm.edu \\ *These authors contributed equally to the manuscript as the first author.}

Received: April 10, 2020

Revised: June 14, 2020

Accepted: June 22, 2020
Copyright $\odot 2020$ Korean Society of Interventional Neuroradiology

This is an Open Access article distributed under the terms of the Creative Commons Attribution Non-Commercial License (http://creativecommons.org/licenses/by-nc/4.0) which permits unrestricted non-commercial use, distribution, and reproduction in any medium, provided the original work is properly cited. 


\section{INTRODUCTION}

Over the past several decades, there has been an expansion of the development and application of minimally invasive endovascular therapies for the treatment of intracranial pathologies. ${ }^{1-5}$ Particularly, there is a growing interest in direct intra-arterial (IA) therapy delivery for central nervous system disorders, a strategy that uses endovascular catheters to deliver therapeutic agents to selected cerebral arterial territories, maximizing the local dose while minimizing the systemic dose. To support this expanding application, a reliable translational model is needed. Canines as animal models for cerebrovascular research exhibit a number of favorable traits. They are reliable under anesthesia, have excellent long-term survival, and possess vessel sizes comparable to humans. ${ }^{6}$ As such, canines have been used as models for the testing and treatment of cerebrovascular diseases. ${ }^{2,6-13}$

Although descriptions of canine cerebral arterial supply and cranial blood flow exists, ${ }^{6}$ there is no singular resource that provides a complete and detailed description of canine cerebral vasculature and there is limited data regarding the feasibility of endovascular cerebral vasculature access for selective IA therapy delivery. During a parallel study focusing on the in-vivo testing of a novel IA cerebral glioma therapy, ${ }^{14}$ we sought to determine the feasibility of transarterial access to the canine cerebral circulation and the ability to perform selective and superselective catheterization of these vessels while obtaining detailed angiographic images and measurements of the canine cerebral vasculature. We report our experience catheterizing the canine cerebral circulation, describe the associated challenges, report normal canine angiographic anatomy, flow patterns, and provide measurements of salient endovascular conduits and intracranial large vessels.

\section{MATERIALS AND METHODS}

\section{Imaging protocol}

All angiographic procedures were performed at the Neurosurgical Education and Innovation Laboratories at Baylor College of Medicine (BCM) with a Siemens Artis Zeego
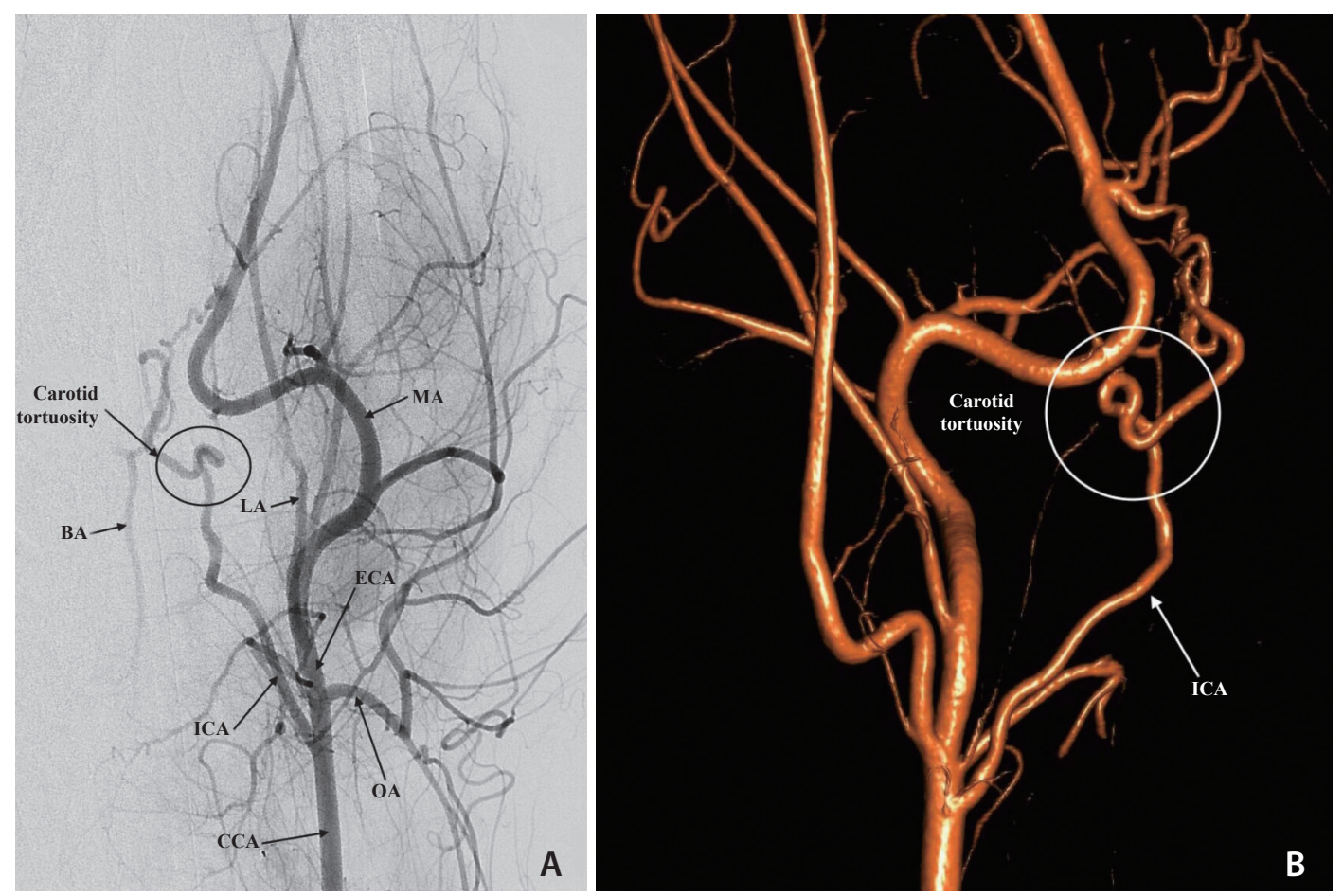

Fig. 1. (A) Digital subtraction angiogram via the left common carotid artery (CCA), anteroposterior view. The distal CCA, external (ECA) and internal carotid (ICA) arteries, occipital artery (OA), lingual artery (LA), and maxillary artery (MA) are opacified. Partial filling of the basilar artery (BA) is observed. The severe tortuosity of the left internal carotid artery is also shown. (B) Three-dimensional rotational carotid angiogram, lateral left view highlights the internal carotid artery (ICA) and the severe tortuosity of the carotid artery. 
monoplane system. The procedures described in this manuscript were reviewed and approved by the Institutional and Animal Care and Use Committee at BCM prior to initiation of experiments and were completed in accordance with federal policies and guidelines (approval number: IACUC protocol \#6339). Three-dimensional (3D) angiographic images were acquired using a 10-second rotational DR acquisition program (syngo DynaCT, Siemens AG). This fast acquisition protocol consists of 250 projections acquired at an angular increment of 0.8 degrees/frame in 10 seconds with a 200-degree angular coverage around the subject. ${ }^{15}$ For all DynaCT scans, 50\% iodinated contrast was injected at a rate of $3 \mathrm{~mL} / \mathrm{s}$ using an automatic power injector, with an imaging acquisition delay of 2 seconds. Breathing was briefly suspended for all scans. All 3D reconstructions were performed on the Siemens syngo Workplace. Two-dimensional (2D) angiographic images were acquired using manual injections of $50 \%$ contrast and a frame rate of 7.5 frames per second. Measurements of the arterial conduits were performed using Siemens Artis Zeego workstation with standard calibration methods. Arterial conduit measurements were performed using the calibrated Siemens workplace. Flow characteristics of the cerebral circulation were determined by reviewing microangiogram runs from various points in the cervical and cerebral vasculature.

\section{Animals and procedures}

Male intact purpose-bred hounds (26 to $35 \mathrm{~kg}$ ) with an average age of 9 months and 15 days, received at least a 5-day acclimation period prior to any procedure. All animals in this study $(n=10)$ were deemed healthy on intake by the BCM veterinary staff. All primary operators are fellowship-trained physicians with over 10 years of experience with neuroendovascular procedures.

In preparation for the procedure, morphine (0.2 to $0.5 \mathrm{mg} / \mathrm{kg}$ ) (Hospira, Lake Forest, IL, USA) in combination with midazolam (0.1 to $0.3 \mathrm{mg} / \mathrm{kg}$ ) (Akorn Pharmaceuticals, Lake Forest, IL, USA) and atropine (0.04 mg/kg) (Vedco Inc., St. Joseph, MO, USA) were administered subcutaneously for initial sedation and prevention of bradycardia. Animals that underwent an excitatory or dysphoric phase also received acepromazine (0.02 to 0.04 mg/kg) (Henry Schein Animal Health, Dublin, $\mathrm{OH}, \mathrm{USA}$ ) intravenously. Propofol (2 to $4 \mathrm{mg} / \mathrm{kg}$ ) (Propoflo; Zoetis, Parsipanny-Troy Hills, NJ, USA) was administered for anesthesia induction. All canines were intubated and anesthesia was maintained using isofluorane (1.5\% to $3.5 \%)$
(Forane; Baxter, Deerfield, IL, USA) delivered in 100\% oxygen. At the end of the study, all animals were euthanized with a commercial phenytoin/pentobartital veterinary euthanasia solution (1 mg/4.5 kg IV) (Beauthanasia-D; Patterson Veterinary, Greeley, CO, USA) followed by necropsy.

Using standard palpation and anatomic landmarks, a micropuncture was performed to access the right femoral artery and a 6-Fr sheath was introduced using a modified Seldinger technique. Heparin (1,000 $\mathrm{U}$ in $1 \mathrm{~L}$ saline) was continuously infused through all guide catheters and microcatheters during the procedures. For angiographic imaging, contrast (Omnipaque 300, lohexol; GE Healthcare, Princeton, NJ, USA) was infused and a single plane digital subtraction angiography (DSA) (see description under Imaging Protocol) was performed. Under fluoroscopic and roadmap guidance, a 6-Fr angled guide catheter, Envoy (Cordis) or

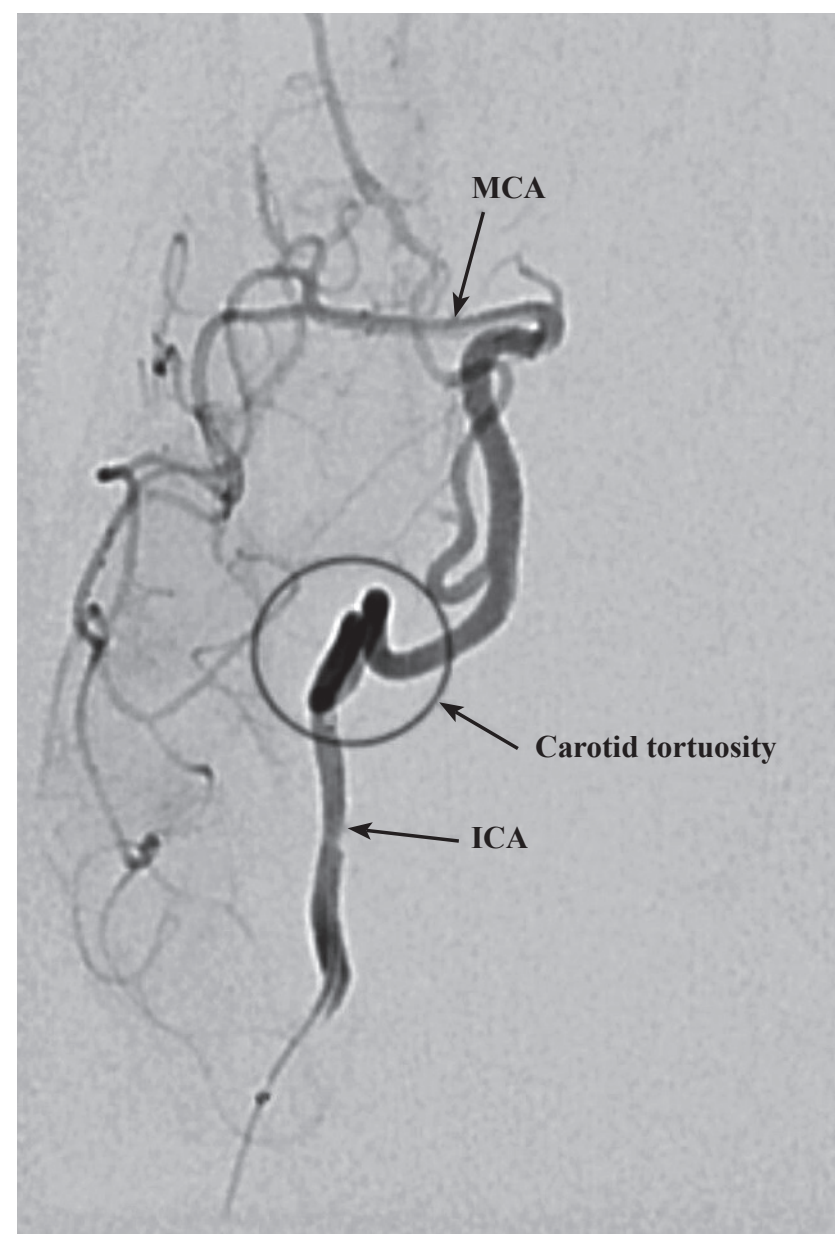

Fig. 2. Selective subtracted angiogram of the right internal carotid artery (ICA), anteroposterior view. A microcatheter injection at the ICA highlights the severe tortuosity of the carotid artery. MCA, middle cerebral artery. 
Chaperon (Microvention Inc., Aliso Viejo, CA, USA), was advanced over an 0.035-in Glidewire (Terumo IS, Somerset, $\mathrm{NJ}$, USA) into the aortic arch and then into the right and left common carotid arteries (CCA) and imaging performed (Fig. 1). A 6-Fr pig tail catheter (Cordis) was used to perform angiography of the aortic arch, great vessels, distal aorta, iliac arteries and surrounding vessel. A 6-Fr guide catheter system was decided upon based on our experiences catheterizing this model in our preliminary studies.

Once in the cervical vessels, a coaxial system comprised
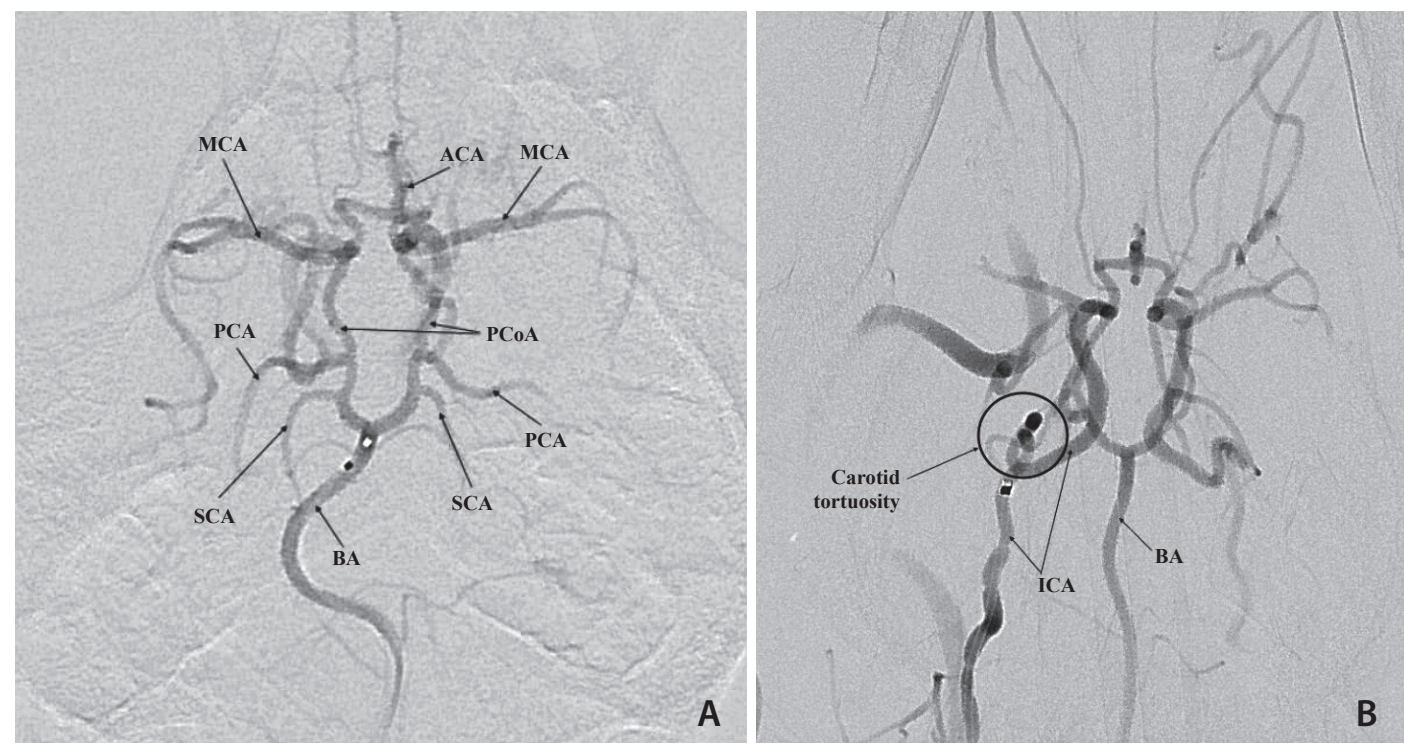

Fig. 3. Selective digital subtraction angiogram of a microcatheter injection of the basilar artery (BA). The entire Circle of Willis (CoW) is visualized in this anteroposterior view. (A) A flow directed microcatheter injection in the distal the BA shows filling of the superior cerebellar arteries (SCA), posterior cerebral arteries (PCA), posterior communicating arteries (PCoA), middle cerebral arteries (MCA), and anterior cerebral arteries (ACA). Note that the BA maintains its natural curve. (B) A guide catheter injection in the right internal carotid artery (ICA) shows filling of the CoW and highlights the severe tortuosity of the carotid artery.
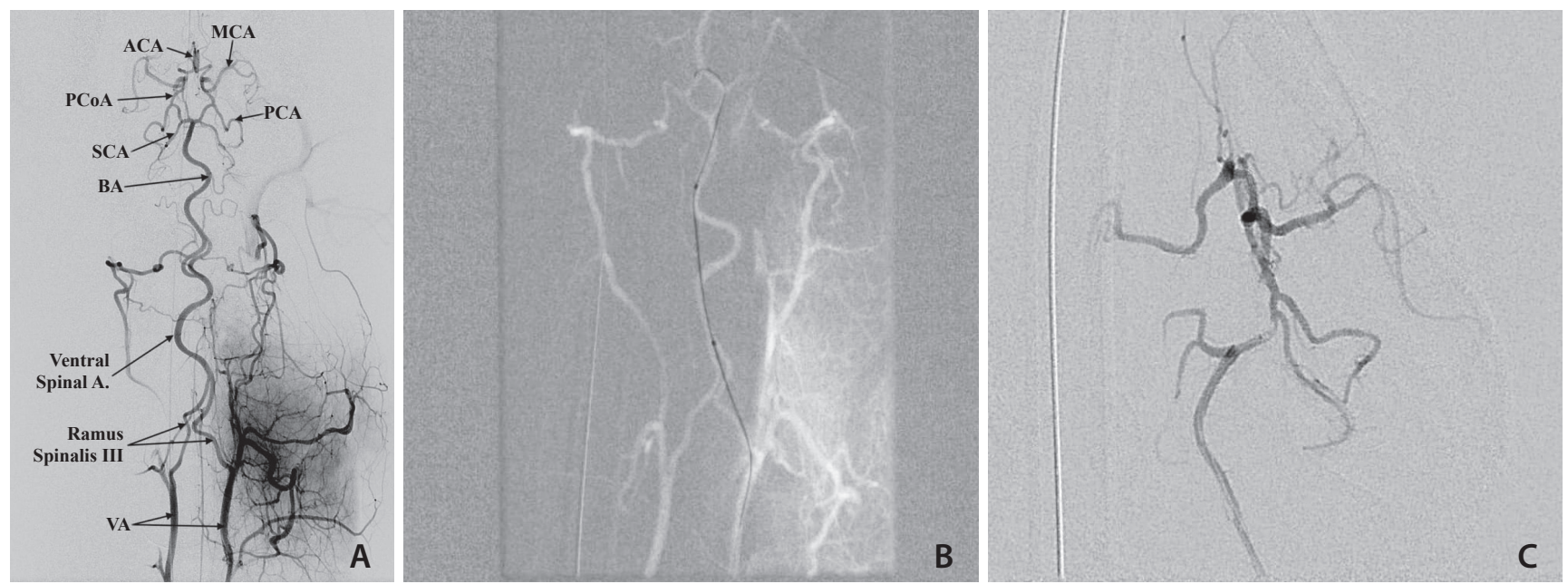

Fig. 4. (A) Digital subtraction angiogram, selective vertebral artery (VA) injection, anteroposterior view. A microcatheter injection at the left VA show fillings of both VA's, the ventral spinal artery, and the basilar artery (BA) and the Circle of Willis (CoW). (B) Roadmap image of the Echelon 10 microcatheter advanced from the Ramus Spinalis to the Ventral Spinal artery and resultant artery straightening secondary to microcatheter stiffness. (C) microcatheter injection in the proximal left P1 segment demonstrating Echelon 10 microcatheter in the cerebral circulation but with significant straightening of the basilar artery. MCA, middle cerebral artery; ACA, anterior cerebral artery; PCoA, posterior communicating artery; SCA, superior cerebellar artery; PCA, posterior cerebral artery. 
of a microwire (Synchro-2 [Stryker, Fremont, CA, USA],Synchro-10 [Stryker] or mirage microwire [Medtronic, Minneapolis, MN, USA]) and microcatheter (Echelon-14 [Medtronic], Marathon [Medtronic], and Magic [Bard, Murray Hill, NJ, USA]) was then established. The coaxial system was used to catheterize the right and left internal carotid arteries (ICA) (Fig. 2), the basilar artery (Fig. 3), vertebral arteries (Fig. 4), and the posterior cerebral arteries (PCA) (Figs. 2, 3A, and 4). 2 and $3 \mathrm{D}$ angiography were performed. For cases of severe arterial vasospasm, additional Verapamil (5 mg; Henry Schein Animal Health) was given intra-arterially just proximal to the site of vasospasm. Microangiograms were performed in each vessel catheterized and studied for downstream flow patterns.

For closure of the recovery animals, a 6-F vascular closure device (Angio-Seal; Terumo IS) was used to seal the right femoral artery and the skin was sutured closed. Manual pressure was then applied to the puncture site for 10 to 15 minutes and followed by application of a local $2 \%$ lidocaine block. Minor complications for recovery animals included non-progressive, minor hematoma formation and slight stridor on recovery due to an elongated soft palate, which were treated with cold compress and supplemental oxygen, respectively. No other significant abnormalities were noted.

\section{RESULTS}

\section{Cerebral arterial catheterization}

Selective and superselective angiography was successful in all subjects with no major IA complications. Precise measurements of key arteries were made from the 2D calibrated angiograms and are listed in Table 1. The mean animal weight was $30.3 \mathrm{~kg}$.

Similar to humans, the canine common femoral artery was found to be very superficial and access was easily achieved by palpation and direct puncture in all subjects $(n=10)$. There was no need for a femoral cut-down or ultrasound guidance, which are often required for other large animal models such as rabbits and swine. ${ }^{15-17}$

Catheter-related vasospasm was seen in all 10 subjects while catheterizing the CCA and vertebral arteries. Vasospasm ranged in severity from mild to severe and was alleviated with a continuous IA infusion of Verapamil $(10 \mathrm{mg}$ in $1 \mathrm{~L}$ saline) through the guide catheter. Extended manipulation and catheter positioning in smaller distal vessels resulted in increased spasm. To ensure the accuracy of vessel measurements, spasms were alleviated prior to measuring vessels.

We were unable to advance the microcatheter into the intracranial circulation via the trans-cervical ICA route in any subject ( $n=7$ ) due to the presence of the severe tortuosity of the carotid artery (Figs. 1, 2, 3B) and reactive arterial vasospasm. This tight turn in the ICA as it passes through the skull base has been noted in other studies as well. ${ }^{18}$ Injections that were performed via a microcatheter positioned in the distal ICA still showed poor antegrade intracranial middle cerebral artery (MCA) and PCA opacification due to competitive flow from the posterior circulation via Circle of Willis interconnections. In all $(n=3)$ subjects tested, injection via the vertebral or basilar arteries demonstrated strong opacification of the MCA and PCA, suggesting that in contrast to humans, the vertebrobasilar system in canines is the dominant source of cerebral large vessel flow (Fig. 3). In 3/3 subjects, we were able to pass the microcatheter via the vertebrobasilar system into the PCA. In the first subject in which posterior circulation was accessed using the larger diameter Echelon microcatheter, there was significant straightening of the vertebrobasilar arteries and we felt that it was unsafe to advance the micro-

Table 1. Diameter measurements of key vessels highlighted in our angiograms

\begin{tabular}{lcc}
\hline Vessel & Size $(\mathrm{mm})$ & Subjects measured $(\mathrm{n})$ \\
\hline FA & $4.64 \pm 0.35$ & 3 \\
IA & $6.65 \pm 0.11$ & 3 \\
\hline Aorta* $^{*}$ & $9.38 \pm 1.12$ & 2 \\
CCA & $4.52 \pm 0.24$ & 7 \\
ECA & $3.65 \pm 0.15$ & 7 \\
ICA & $1.60 \pm 0.18$ & 8 \\
BA & $1.65 \pm 0.19$ & 4 \\
VA & $1.53 \pm 0.05$ & 2 \\
P1 & $1.23 \pm 0.11$ & 4 \\
P2 & $1.16 \pm 0.17$ & 4 \\
PCOM & $1.15 \pm 0.09$ & 4 \\
MCA & $1.49 \pm 0.33$ & 6 \\
\hline Va & m a & 4 \\
\hline
\end{tabular}

Values are presented as mean \pm standard deviation or number only. Average subject weight: $30.3 \mathrm{~kg}$, subject weight range: 26 to $35 \mathrm{~kg}$.

FA, femoral artery; IA, iliac artery; CCA, common carotid artery; ECA, external carotid artery; ICA, internal carotid artery; BA, basilar artery; VA, vertebral artery; P1 \& P2, segments of posterior cerebral artery; PCOM, posterior communicating artery; MCA, middle cerebral artery.

*Measure at mid thoracic aorta. 
catheter beyond the proximal PCA (Fig. 4). In the 2 subjects where flow-directed catheters were used, the catheter was successfully advanced through the vertebrobasilar system and into the posterior communicating artery (PCOA) (Figs. $5 A-C)$. While the PCA and PCoA's were accessible with flow directed catheters and the posterior circulation branches readily catheterized (Figs. 5D-F), intracranial tortuosity made advancement across the posterior circulation into the anterior circulation via the PCoA challenging. Since we were aiming access the Circle of Willis but avoid subarachnoid hemorrhage (SAH), we chose not to risk cerebral vessel injury by making the vertical turn into the ICA.

Post-mortem analyses demonstrated SAH in 2 of the 10 canines. In both cases, the perivascular hemorrhage surrounded the basilar artery and extended cranially along the PCA and PCOA. The SAH were experienced in 2/3 animals where the vertebrobasilar system was accessed with Echelon 10 catheters, and the bleed was most likely caused by intra-procedural vascular displacement during microwire and microcatheter passage through the curvature or the basilar artery to the intracranial posterior circulation (Fig. 4). In the animal where trans-vertebrobasilar cerebral circulation access was solely attempted with flow directed microcatheter (Magic), there was no SAH. In the canines with internal carotid access attempts only, there was no SAH. Cerebral blood flow patterns were documented based on microangiogram
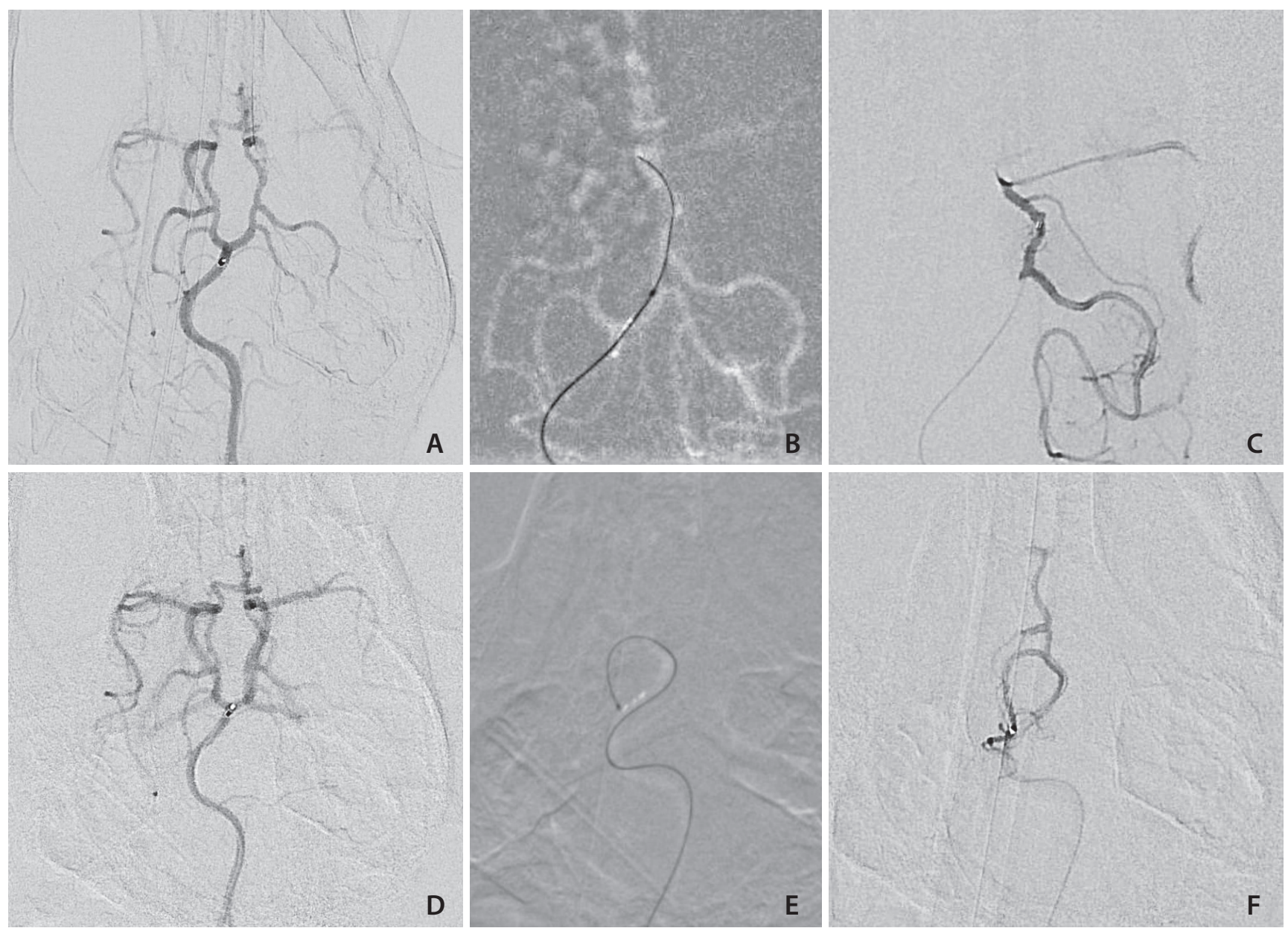

Fig. 5. (A) Anterior-posterior digital subtraction angiography (DSA) angiogram of the basilar artery and Circle of Willis. The flow directed marathon catheter tip is at the basilar artery bifurcation without deforming the natural basilar artery curvature. (B) Roadmap demonstrating the flowdirected microcatheter being advanced over the wire to the posterior communicating artery) PCoA: carotid junction. (C) Microcatheter contrast injection DSA demonstrating the catheter near the PCoA: carotid artery junction. (D) Pre-catheterization posterior circulation angiogram demonstrating the flowdirected microcatheter in the distal basilar. (E) Road map image showing the flowdirected microcatheter advanced into the right superior cerebellar artery (SCA), making a >180 degree turn without deforming the arterial tree. (F) Superselective contrast injection into the SCA with some reflux into the right $\mathrm{PCOA}$. 
performed in each vessel catheterized (Table 2).

The imaging demonstrated that the posterior circulation is dominant and fills the PCAs, MCAs, and anterior cerebral arteries (ACAs) bilaterally with even a modest injection rate. Injection in the cervical ICA filled ipsilateral MCA and ACA. When the ipsilateral PCoA was injected, the ipsilateral PCA, MCA and the ACA filled.

Angiographic arterial conduits that are relevant for neuroendovascular access and treatment planning were measured in all animals with suitable injections. The femoral artery was found to have a mean diameter of $4.64 \mathrm{~mm}$, which was large enough to accommodate $6-\mathrm{F}$ sheaths that are used in humans and an Angio-Seal closure device without difficulty or complication. The iliac artery and mid-thoracic aorta measured $6.65 \mathrm{~mm}$ and $9.38 \mathrm{~mm}$, respectively (Table 1). The external carotid artery was found to have a mean diameter $3.65 \mathrm{~mm}$ while the ICA diameter was measured to be 1.60 $\mathrm{mm}$. Notable intracranial vessel diameters include the basilar artery, which had a comparable diameter to the ICA. The Circle of Willis was noted to be robust with $1.15 \mathrm{~mm}$ diameter PCoA's leading to robust opacification of the MCAs $(1.49 \mathrm{~mm})$ via basilar artery injection.

\section{DISCUSSION}

In this present study, we report our experience catheterizing canine cerebral circulation with endovascular microcatheters, present high-resolution angiographic images of the canine vascular anatomy spanning the femoral arteries through the Circle of Willis, describe arterial branch flow patterns and real-time cerebral circulation flow characteristics and provide measurements of canine arterial conduits for endovascular procedure planning.

In doing so, we found that the canine arterial anatomy accommodated cervical ICA catheterization, but did not accommodate further microcatheter advancement into intracranial vessels due to a combination of small vessel diameter, significant tortuosity of the carotid artery and severe catheter related vasospasm. Based on angiography contrast flow, IA therapy administered into the cervical ICA will perfuse the ipsilateral anterior circulation (Fig. 2), but is less selective than direct intracranial branch catheterization. The Circle of Willis and its posterior branches were accessible through the vertebrobasilar system, which on angiography was the dominant source of Circle of Willis flow. Based on angiographically observed flow patterns, basilar artery IA infusions will flow into the posterior and anterior circulations simultaneously and bilaterally (Figs. 3, 4), an ipsilateral PCA and MCA simultaneously via unilateral PCA infusion, or in a superselective manner into either PCA or SCA with superselective catheterization (Fig. 5, Table 2). Our experience supported the use of flow directed microcatheters like Marathon and Magic to access the Circle of Willis and its branches via the vertebrobasilar system due to smaller catheter diameter and increased flexibility allowing greater conformity to the natural posterior circulation arterial curvature.

Challenges encountered while accessing the canine cervical and cerebral circulation included catheter related local arterial vasospasm, which was particularly severe when attempting to pass through the tortuous ICA. Similar to humans, canine vasospasm was effectively resolved with IA Verapamil plus time and there was an expected modest drop in blood pressure. ${ }^{10}$ Care is still warranted during administration of this drug to prevent inadvertent toxicity leading to bradyarrhythmia and heart block, especially when pre-loading prior to initiation of CRI administration. Particularly when used in the posterior circulation, we found that the standard human endovascular microcatheters SL-10 and Echelon-10 were rigid compared to the flow directed catheters and advancing them beyond the basilar apex straightened the natural curvature and risked vessel injury. The size and rigidity of the standard microcatheters passing through the vertebrobasilar

\section{Table 2. Microangiogram: cerebral arterial flow patterns}

\begin{tabular}{llc}
\hline Vessel injected & \multicolumn{1}{c}{ Territory visualized } & Reliability \\
\hline ICA & MCA (ipsilateral) & +++ \\
& ACA (ipsilateral) & + \\
Basilar artery & SCA (bilateral) & +++ \\
& PCA (bilaterally) & +++ \\
& PCOA (bilaterally) & +++ \\
& MCA (bilaterally) & ++ \\
& ACA (bilaterally) & + \\
PCoA & PCA (ipsilateral) & +++ \\
& MCA (ipsilateral) & +++ \\
& ACA (ipsilateral) & ++ \\
& ACA (contralateral) & + \\
PCA (superselective) & PCA (ipsilateral) & ++++ \\
& PCOA (ipsilateral)* & + \\
& MCA (ipsilateral)* & + \\
\hline
\end{tabular}

ICA, internal carotid artery; MCA, middle cerebral artery; ACA, anterior cerebral artery; SCA, superior cerebellar artery; PCA, posterior cerebral artery; PCoA, posterior communicating artery. ${ }^{*}$ Reflux; ${ }^{\dagger}$ Circle of Willis crossflow. 
is believed to have led to SAH in the two subjects. In addition to better navigability, flow directed catheters minimized displacement of the vertebrobasilar arteries on angiography. The smaller inner lumen of flow directed catheter might limit some endovascular procedures, but was not found to negatively impact IA visualization during microcatheter contrast injections nor impede cellular therapies in an in vitro study of stem cell infusion and will easily accommodate IA chemotherapy infusions.

A variety of large animal species have been utilized as pre-clinical models for cerebrovascular disease therapy testing, particularly for stroke research, including ovis, swine, felines, leporidae and non-human primates. ${ }^{6}$ Smaller animals such as rodents, felines and leporidae have arterial systems too small for direct cerebral catheterization. Canines have favorable arterial anatomy, a gyrencephalic brain with grey/ white matter similar to humans and lack a cervical arterial rete mirabile (present in swine). ${ }^{6}$ Other groups have developed endovascular permanent MCA occlusion stroke models in canines via thrombus injection into the cervical ICA and distal embolization to the target vessel. ${ }^{7,18,19}$ More recently, Zhang et al. ${ }^{11}$ developed a canine posterior circulation stroke model for testing mechanical thrombectomy devices in the basilar artery. Two prior studies have described accessing the cerebral circulation with microcatheters for the purpose of creating a reversible MCA occlusion stroke model. ${ }^{20,21}$ Both groups successfully accessed the Circle of Willis via the vertebrobasilar system with SL-10 microcatheters, reached the carotid terminus via the PCOA and advanced a coil in the carotid terminus/MCA to induce an ischemic stroke. The current study confirmed their experience catheterizing the canine the cerebral circulation, and further, we found that there was less vessel deformation and better navigability when using next generation flow directed microcatheters catheters to access the Circle of Willis. Finally, even very small canine cerebral branches can be safely superselected with the flow directed microcatheters which is critical for many IA therapy paradigms in humans.

There are currently a number of ongoing cerebral disorder treatments being investigated with IA delivery strategies, most notably cerebral malignancy and ischemic stroke. IA delivery of chemotherapeutic agents may allow for blood brain barrier disruption and delivery of increased therapy concentrations locally, while sparing toxic doses to the systemic circulation. ${ }^{22,23}$ A variety of animal models have been utilized in the preclinical testing of IA stem cell infusion after acute ischemic stroke and phase I and phase II trials in humans subjects are ongoing. ${ }^{2,3}$ Further, this canine IA therapy model is being investigated in preparation for future testing of a novel glioblastoma, stem cell loaded with a tumor targeted viral vector. ${ }^{14}$ The results of canine our stem cell safety and dosing experiments as well as post-procedure pathology and imaging will be reported subsequently.

To our knowledge, this study represents the most comprehensive canine angiographic vascular anatomy in the literature We hope our findings and experiences will aid in the design of future studies and reduce the use of resources for preliminary testing of animal models.

\section{CONCLUSION}

The canine model is favorable for cerebral IA therapy testing with the potential for superselective cerebral branch IA infusion. The intracranial vasculature was best accessed with flow directed microcatheters via the dominant vertebrobasilar circulation and the anterior circulation could be accessed in a less selective manner via the cervical ICA. It is feasible to deliver IA transcatheter therapies to selective vascular territories within the canine cerebral circulation, thus making it a viable animal model for testing novel IA cerebral treatments.

\section{Study limitations}

This study has some important study limitations. Since the purposes of the parallel study did not require visualization of the femoral and iliac arteries, there is a low sample size $(n=2)$ for measurements of these vessels. It is the nature of the primary study that led to a smaller sample size, not the lack of accessibility of the vessels. Similarly, with regards to the lower sample sizes $(n=2)$ for measurements of both the aorta and vertebral arteries, as many measurements of these vessels were taken in the context of the primary study.

\section{Acknowledgments}

We would like to thank Dr. Dalis Collins and her veterinary care team from the Center for Comparative Medicine at Baylor College of Medicine for their exceptional care of our animal subjects.

\section{Fund}

None. 


\section{Ethics Statement}

All procedures described in this manuscript were reviewed and approved by the Institutional and Animal Care and Use Committee (IACUC) at Baylor College of Medicine prior to initiation of experiments and were completed in accordance with federal policies and guidelines.

\section{Conflicts of Interest}

Dr. Peter Kan is a consultant for Stryker Neurovascular and Medtronic. The other authors have no conflicts to disclose.

\section{Author Contributions}

Conception and design: KMC, VMS, PK, SC, and JJ. Acquisition of data: all authors. Analysis and interpretation of data: KMC, VMS, and JJ. Drafting the article: KMC, VMS, DC, and JJ. Reviewed submitted version of article: all authors. Statistical analysis: KMC. Study supervision: DC, PK, SC, and JJ.

\section{ORCID}

Kevin M. Camstra: https://orcid.org/0000-0003-1726-5980

Visish Srinivasan: https://orcid.org/0000-0001-7673-6805

Dalis Collins: https://orcid.org/0000-0003-2484-1640

Stephen Chen: https://orcid.org/0000-0002-5580-831X

Peter Kan: https://orcid.org/0000-0003-4839-876X

Jeremiah Johnson: https://orcid.org/0000-0002-9920-5895

\section{REFERENCES}

1. Guzman R, Janowski M, Walczak P. Intra-arterial delivery of cell therapies for stroke. Stroke 2018:49:1075-1082

2. Misra V, Lal A, El Khoury R, Chen PR, Savitz SI. Intra-arterial delivery of cell therapies for stroke. Stem Cells Dev 2012;21:1007-1015

3. Moniche F, Escudero I, Zapata-Arriaza E, Usero-Ruiz M, Prieto-León M, de la Torre J, et al. Intra-arterial bone marrow mononuclear cells (BM-MNCs) transplantation in acute ischemic stroke (IBIS trial): protocol of a phase II, randomized, dose-finding, controlled multicenter trial. Int J Stroke 2015;10:1149-1152

4. Silachev DN, Plotnikov EY, Babenko VA, Danilina TI, Zorov LD, Pevzner IB, et al. Intra-arterial administration of multipotent mesenchymal stromal cells promotes functional recovery of the brain after traumatic brain injury. Bull Exp Biol Med 2015;159:528533

5. Spiliopoulos S, Festas G, Theodosis A, Palialexis K, Reppas L, Konstantos $C$, et al. Incidence and endovascular treatment of severe spontaneous non-cerebral bleeding: a single-institution experience. Eur Radiol 2019;29:3296-3307

6. Atchaneeyasakul K, Guada L, Ramdas K, Watanabe M, Bhattacharya P, Raval AP, et al. Large animal canine endovascular ischemic stroke models: a review. Brain Res Bull 2016;127:134140

7. Bouzeghrane F, Naggara O, Kallmes DF, Berenstein A, Raymond J; International Consortium of Neuroendovascular Centres. In vivo experimental intracranial aneurysm models: a systematic review. AJNR Am J Neuroradiol 2010;31:418-423

8. Raymond J, Guilbert F, Metcalfe A, Gévry G, Salazkin I, Robledo O. Role of the endothelial lining in recurrences after coil embolization: prevention of recanalization by endothelial denudation. Stroke 2004;35:1471-1475

9. Shin YS, Niimi Y, Yoshino Y, Song JK, Silane M, Berenstein A. Creation of four experimental aneurysms with different hemodynamics in one dog. AJNR Am J Neuroradio/ 2005;26:1764-1767

10. Tilley L, Smith F, Oyama M, Sleeper M. Manual of canine and feline cardiology, 4th ed. St Louis: Saunders; 2008

11. Zhang $Y$, Jin $M, D u B$, Lin $H, X u C$, Jiang $W$, et al. A novel canine model of acute vertebral artery occlusion. PLoS One 2015;10:e0142251

12. Mawad ME, Mawad JK, Cartwright J Jr, Gokaslan Z. Long-term histopathologic changes in canine aneurysms embolized with Guglielmi detachable coils. AJNR Am J Neuroradiol 1995;16:7-13

13. Zu QQ, Liu S, Xu XQ, Lu SS, Sun L, Shi HB. An endovascular canine stroke model: middle cerebral artery occlusion with autologous clots followed by ipsilateral internal carotid artery blockade. Lab Invest 2013;93:760-767

14. Lang FF, Conrad C, Gomez-Manzano C, Yung WKA, Sawaya R, Weinberg JS, et al. Phase I study of DNX-2401 (delta-24-RGD) oncolytic adenovirus: replication and immunotherapeutic effects in recurrent malignant glioma. J Clin Oncol 2018;36:14191427

15. Srinivasan VM, Chintalapani G, Camstra KM, Effendi ST, Cherian J, Johnson JN, et al. Fast acquisition cone-beam computed tomography: initial experience with a $10 \mathrm{~S}$ protocol. J Neurointerv Surg 2018;10:916-920

16. Cloft HJ, Altes TA, Marx WF, Raible RJ, Hudson SB, Helm GA, et al. Endovascular creation of an in vivo bifurcation aneurysm model in rabbits. Radiology 1999;213:223-228

17. Wallace MJ, Ahrar K, Wright KC. Validation of US-guided percutaneous venous access and manual compression for studies in swine. J Vasc Interv Radiol 2003;14:481-483

18. Boulos AS, Deshaies EM, Dalfino JC, Feustel PJ, Popp AJ, Drazin D. Tamoxifen as an effective neuroprotectant in an endovascular canine model of stroke. J Neurosurg 2011;114:1117-1126 
19. Lu SS, Liu S, Zu QQ, Xu XQ, Yu J, Wang JW, et al. In vivo MR imaging of intraarterially delivered magnetically labeled mesenchymal stem cells in a canine stroke model. PLoS One 2013;8:e54963

20. Christoforidis GA, Rink C, Kontzialis MS, Mohammad Y, Koch RM, Abduljalil AM, et al. An endovascular canine middle cerebral artery occlusion model for the study of leptomeningeal collateral recruitment. Invest Radio/ 2011;46:34-40

21. Rink C, Christoforidis G, Abduljalil A, Kontzialis M, Bergdall V, Roy $\mathrm{S}$, et al. Minimally invasive neuroradiologic model of preclinical transient middle cerebral artery occlusion in canines. Proc Natl Acad Sci U S A 2008;105:14100-14105

22. Ellis JA, Cooke J, Singh-Moon RP, Wang M, Bruce JN, Emala CW, et al. Safety, feasibility, and optimization of intra-arterial mitoxantrone delivery to gliomas. J Neurooncol 2016;130:449-454

23. Peschillo S, Caporlingua A, Diana F, Caporlingua F, Delfini R. New therapeutic strategies regarding endovascular treatment of glioblastoma, the role of the blood-brain barrier and new ways to bypass it. J Neurointerv Surg 2016;8:1078-1082 